Pacific Journal of Mathematics

A NOTE ON REGULAR CAUCHY SPACES 


\section{A NOTE ON REGULAR CAUCHY SPACES}

\section{C. KENT}

A regular convergence space has both a finest and coarsest compatible regular Cauchy structure. The coarsest compatible regular Cauchy structure is complete if and only if the original space is Urysohn-closed; it is totally bounded if and only if the original space is almost topological. Minimal regular Cauchy spaces are characterized and, in the complete case, shown to be in one-to-one correspondence with the minimal regular convergence spaces. The noncomplete minimal regular regular Cauchy spaces do not have regular completions.

1. Fine and coarse Cauchy structures. The reader is asked to refer to [2] for definitions, notation, and terminology pertaining to convergence and Cauchy spaces not given here. As in [2], we make the assumption that all convergence and Cauchy spaces are Hausdorff.

A few nonstandard notations which we shall borrow from [2] are worth special mention. If filters $\mathscr{F}$ and $\mathscr{G}$ contain disjoint sets,

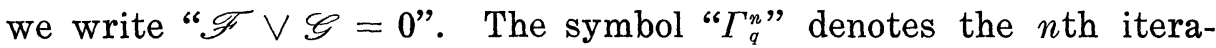
tion of the closure operator with respect to a convergence structure $q$. The term "ultrafilter" will be abbreviated "u.f.".

Given a Cauchy space $(X, \mathscr{C})$, the associated convergence structure is denoted $q_{\mathscr{E}}$; in other words, $\mathscr{F} q_{\mathscr{E}}$-converges to $x$ iff $\dot{x} \cap \mathscr{F} \in \mathscr{C}$.

Let $F(X)$ denote the set of all filters on $X$. Given a convergence space $(X, q)$, let $[q]$ denote the set of all Cauchy structures $\mathscr{C}$ on $X$ such that $q=q_{\mathscr{8}}$. Also associated with $q$ are the following sets of filters:

$$
\begin{aligned}
& \mathscr{C}^{q}=\{\mathscr{F} \in F(X): \mathscr{F} \text { is } q \text {-convergent }\} \\
& \Lambda_{q}=\left\{\mathscr{F} \in F(X): \mathscr{F} \vee \mathscr{G}=0 \text { for all } \mathscr{G} \in \mathscr{C}^{q}\right\} \\
& \mathscr{C}_{q}=\mathscr{C}^{q} \cup \Lambda_{q} .
\end{aligned}
$$

We omit the easy proof of the first proposition.

Proposition 1.1. For any convergence space $(X, q), \mathscr{C}^{q}$ is the finest member of $[q] . \quad \mathscr{C}^{q}$ is regular iff $q$ is regular. $\mathscr{C}^{q}$ is complete.

Proposition 1.2. For any convergence space $(X, q), \mathscr{C}_{q}$ is the coarsest member of $[q] . \quad \mathscr{C}_{q}$ is totally bounded.

Proof. The verification that $\mathscr{C}_{q}$ is a Cauchy structure compatible 
with $q$ is straightforward. If $\mathscr{C} \in[q]$ and $\mathscr{F} \in \mathscr{C}$, then either $\mathscr{F}$ if $q$-convergent, in which case $\mathscr{F} \in \mathscr{C}^{q}$, or else $\mathscr{F}$ is non- $q$-convergent, in which case $\mathscr{F} \vee \mathscr{G}=0$ for all $\mathscr{G} \in \mathscr{C}^{q}$, and hence $\mathscr{F} \in \Lambda_{q}$. In either case, $\mathscr{F} \in \mathscr{C}_{q}$, and so $\mathscr{C}_{q} \leqq \mathscr{C}$. If $\mathscr{H}$ is a non-q-convergent u.f., then $\mathscr{H} \vee \mathscr{G}=0$ for all $\mathscr{G} \in \mathscr{C}^{q}$, and therefore $\mathscr{G} \in \mathscr{C}_{q}$; thus $\mathscr{C}_{q}$ is totally bounded.

For a convergence space $(X, q), \mathscr{C}^{q}$ will be called the fine Cauchy structure and $\mathscr{C}_{q}$ the coarse Cauchy structure associated with $q$.

Theorem 1.3. Let $(X, q)$ be a convergence space. Then $\mathscr{C}_{q}$ is regular iff $q$ is regular and almost topological.

Proof. Assume that $q$ is regular and almost topological. If $\mathscr{F} \in \mathscr{C}^{q}$, then $\Gamma_{q} \mathscr{F} \in \mathscr{C}^{q}$ by Proposition 1.1. If $\mathscr{F} \in \Lambda_{q}$ and $\Gamma_{q} \mathscr{F} \notin \Lambda_{q}$, then there is a filter $\mathscr{G} \ni \mathscr{C}^{q}$ such that $\left(\Gamma_{q} \mathscr{F}\right) \vee \mathscr{G} \neq 0$. Without loss of generality, we can assume that $\mathscr{F}$ is an u.f. Since $(X, q)$ has the same u.f. convergence as a topological space, the point to which $\mathscr{G} q$-converges (call it $x$ ) is an adherent point for $\mathscr{F}$. Under the assumption that $\mathscr{F}$ is an u.f., $\mathscr{F} q$-converges to $x$. But this contradicts the assumption that $\mathscr{F} \in \Lambda_{q}$, and this contradiction shows that $\Gamma_{q} \mathscr{F} \in \Lambda_{q}$. Thus $\mathscr{C}_{q}$ is regular.

Conversely, assume that $\mathscr{C}_{q}$ is regular. Then $q$ is obviously regular, and it remains to show that $q$ is almost topological. First, let $\mathscr{F} \geqq \mathscr{V}_{q}(x)$, where $\mathscr{F}$ is an u.f. and $\mathscr{V}_{q}(x)$ the $q$-neighborhood filter at $x$. Then $\dot{x} \geqq \Gamma_{q} \mathscr{F}$. Since $\mathscr{C}_{q}$ is totally bounded, $\mathscr{F} \in \mathscr{C}_{q}$, and hence $\mathscr{F} q$-converges to $x$. The proof will be completed by showing that the $q$-closure operator is idempotent. Let $x \in \Gamma_{q}^{2} A$. Then there is an u.f. $\mathscr{K} q$-converging to $x$ such that $\Gamma_{q}(A) \in \mathscr{K}$. By Lemma 2.1 of [2], there is an u.f. $\mathscr{H}$ containing $A$ such that $\mathscr{K} \geqq \Gamma_{q} \mathscr{H}$. Since $\dot{x} \geqq \Gamma_{q} \mathscr{K} \geqq \Gamma_{q}^{2} \mathscr{C}$ and $\mathscr{H} \in \mathscr{C}_{q}$, $\mathscr{C} q$-converges to $x$. Therefore, $x \in \Gamma_{q} A$. Since the $q$-closure operator is idempotent, it follows that $(X, q)$ is almost topological, and the proof is complete.

2. The coarsest regular Cauchy structure. Let $N$ denote the set of natural numbers, and let $\Gamma_{q}^{n}$ denote the $n$th iteration of the closure operator of a convergence $(X, q)$. With each regular convergence space $(X, q)$, we shall associate two additional sets of filters defined as follows:

$$
\begin{aligned}
& \Delta_{q}=\left\{\mathscr{F} \in F(X): \mathscr{G} \vee\left(\Gamma_{q}^{n} \mathscr{F}\right)=0 \text { for all } \mathscr{G} \in \mathscr{C}^{q} \text { and } n \in N\right\} . \\
& \mathscr{D}_{q}=\mathscr{C}^{q} \cup \Delta_{q} .
\end{aligned}
$$

Proposition 2.1. If $(X, q)$ is a regular convergence space, then $\mathscr{D}_{q}$ is the coarsest regular member of $[q]$. 
Proof. It is a routine matter to verify that $\mathscr{D}_{q}$ is a Cauchy structure compatible with $q$. To show that $\mathscr{D}_{q}$ is regular, let $\mathscr{F} \in \Delta_{q}$. Since $\left(\Gamma_{n}^{q} \mathscr{F}\right) \vee \mathscr{G}=0$ for all $n$ in $N$ and $\mathscr{G} \in \mathscr{C}^{q}, \Gamma_{q}^{n}\left(\Gamma_{q} \mathscr{F}\right) \vee \mathscr{G}=0$ for all $n$ in $N$ and $\mathscr{G} \in \mathscr{C}^{q}$. Thus $\Gamma_{q} \mathscr{F} \in \Delta_{q}$, and the regularity of $\mathscr{D}_{q}$ is established. An argument like that used in the proof of Proposition 1.2 shows that $\mathscr{D}_{q}$ is the coarsest regular member of $[q]$.

A convergence space $(X, q)$ is said to be a Urysohn space if, whenever $\mathscr{F} q$-converges to $x$ and $\mathscr{G} q$-converges to $y$, for $x \neq y$, it follows that $\Gamma_{q}^{n}(\mathscr{F}) \vee \Gamma_{q}^{n}(\mathscr{G})=0$ for all $n$ in $N$. A regular convergence space is obviously a Urysohn space.

A regular (Urysohn) convergence space is regular-closed (Urysohnclosed) if it is a closed subspace of every regular (Urysohn) convergence space in which it is embedded. Characterizations of regularclosed and Urysohn-closed convergence spaces are given in [1]; these results are restated, in modified form, in the following lemma.

Given a convergence space $(X, q)$ and $\mathscr{F} \in F(X)$, let $\Gamma_{q}^{\omega} \mathscr{F}=$ $\cap\left\{\Gamma_{q}^{n} \mathscr{F}: n \in N\right\}$.

LEMMA 2.2. Let $(X, q)$ be a regular convergence space.

(a) $(X, q)$ is regular-closed iff, for each $\mathscr{F} \in F(X), \Gamma_{q}^{\omega}(\mathscr{F})$ has an adherent point.

(b) $(X, q)$ is Urysohn iff, for each $\mathscr{F} \in F(X)$, there is $n \in N$ such that $\Gamma_{q}^{n}(\mathscr{F})$ has an adherent point.

THEOREM 2.3. A regular convergence space $(X, q)$ has a unique compatible regular Cauchy structure iff $(X, q)$ is regular and Urysohnclosed.

Proof. The set $[q]$ contains a unique regular member iff $\Delta_{q}=\varnothing$. If $\Delta_{q}=\varnothing$, then for each filter $\mathscr{F}$ on $X,\left(\Gamma_{q}^{n} \mathscr{F}\right) \vee \mathscr{G} \neq 0$ for some $n \in N$ and $\mathscr{G} \in \mathscr{C}^{q}$. Thus $\Gamma_{q}^{n} \mathscr{F}$ has an adherent point, and $(X, q)$ is Urysohn-closed by Lemma 2.2. Conversely, assume that $\Delta_{q}$ contains a filter $\mathscr{F}$. Since $\mathscr{G} \vee\left(\Gamma_{q}^{n} \mathscr{F}\right)=0$ for all $\mathscr{G} \in \mathscr{C}^{q}$ and $n \in N, \Gamma_{q}^{n} \mathscr{F}$ has no adherent points for all $n \in N$, and by Lemma $2.2,(X, q)$ is not Urysohn-closed.

From Theorem 2.3 and Lemma 2.2, it follows that every convergence space with a unique compatible regular Cauchy structure is regular-closed.

CoROLlaRY 2.4. Let $(X, q)$ be a regular convergence space.

(a) $\left(X, \mathscr{D}_{q}\right)$ is complete iff $(X, q)$ is Urysohn-closed.

(b) $\left(X, \mathscr{D}_{q}\right)$ is totally bounded iff $(X, q)$ is almost topological.

Proof. (a) Since $\Delta_{q}$ is the set of nonconvergent Cauchy filters 
in $\mathscr{D}_{q}, \mathscr{D}_{q}$ is complete iff $\Delta_{q}=\varnothing$. In the proof of Theorem 2.3, it is shown that $\Delta_{q}=\varnothing$ iff $(X, q)$ is Urysohn-closed.

(b) $\left(X, \mathscr{D}_{q}\right)$ is totally bounded iff $\mathscr{D}_{q}=\mathscr{C}_{q}$. The latter condition is, by Theorem 1.3 and Proposition 2.1, equivalent to $(X, q)$ being almost topological.

If $(X, q)$ is a regular convergence space which is not Urysohnclosed, then let $\mathscr{M}_{q}$ be the filter obtained by intersecting all of the filters in $\Delta_{q}$.

THEOREM 2.5. If $(X, q)$ is a regular convergence space which is not Urysohn-closed, then $\left(X, \mathscr{D}_{q}\right)$ has a regular completion iff $\mathscr{F} \vee \mathscr{M}_{q}=0$ for all $\mathscr{F} \in \mathscr{C}^{q}$.

Proof. The set $\Delta_{q}$ constitutes the single equivalence class of nonconvergent Cauchy filters in $\mathscr{D}_{q}$. Thus any completion of $\left(X, \mathscr{D}_{q}\right)$ is necessarily a one-point completion, with all filters in $\Delta_{q}$ converging in the completion space to the added point.

Assume that $(Y, \mathscr{C})$ is a regular one-point completion of $\left(X, \mathscr{D}_{q}\right)$. Without loss of generality, we can let $Y=X \cup\{a\}$, where $a \notin X$, and consider $\left(X, \mathscr{D}_{q}\right)$ as a subspace of $(Y, \mathscr{C})$. If $\mathscr{F} \in \mathscr{C}^{q}$ and $\mathscr{F} \vee \mathscr{M}_{q} \neq 0$, then there is an u.f. $\mathscr{G} \geqq \mathscr{F}$ such that $\mathscr{G} \geqq \mathscr{M}_{q}$. Thus every set $G$ in $\mathscr{G}$ belongs to an u.f. in $\Delta_{q}$, and therefore $\dot{a} \geqq \Gamma_{q_{\mathscr{S}}} \mathscr{G}^{\prime}$, where $\mathscr{G}^{\prime}$ is the filter on $Y$ generated by $\mathscr{G}$. But $\mathscr{G}^{\prime}$ also $q_{\mathscr{E}}$-converges to some element in $X$, and it follows that $(Y, \mathscr{C})$ cannot be both regular and Hausdorff. This contradiction establishes that $\mathscr{F} \vee \mathscr{M}_{q}=0$ for all $\mathscr{F} \in \mathscr{C}^{q}$.

Conversely, assume that $\mathscr{F} \vee \mathscr{C}_{q}=0$ for all $\mathscr{F} \in \mathscr{C}^{q}$. Let $Y=X \cup\{a\}$, where $a \notin X$, and let $\mathscr{C}$ be the canonical one-point completion Cauchy structure in which the filters in $\Delta_{q}$, considered as filters on $Y$, converge to $a$. One can readily verify that the assumed condition is precisely what is needed to establish that $(Y, \mathscr{C})$ is regular.

3. Minimal regular Cauchy spaces. A regular convergence space $(X, q)$ is minimal regular if, whenever $p$ is a regular convergence structure on $X$ and $p \leqq q$, then $p=q$. A characterization of minimal regular convergence spaces, which we shall not make use of, is given in [1]. A minimal regular Cauchy space is defined in the analogous (and obvious) way.

TheOREM 3.1. A complete Cauchy space $(X, \mathscr{C})$ is minimal regular iff $\left(X, q_{\mathscr{E}}\right)$ is a minimal regular convergence space.

Proof. Let $(X, \mathscr{C})$ be a complete minimal regular Cauchy space. 
If $q=q_{\mathscr{E}}$, then $\mathscr{C}=\mathscr{C}^{q}$ by the assumption that $(X, \mathscr{C})$ is complete. If there is a regular convergence structure $p \leqq q$, then $\mathscr{C}^{p} \leqq \mathscr{C}^{q}$, and so by assumption $\mathscr{C}^{p}=\mathscr{C}^{q}$. But this implies $p=q$, and so $(X, q)$ is a minimal regular convergence space.

Conversely, if $q$ is minimal regular and $\mathscr{C}^{q}=\mathscr{C}$, then let $\mathscr{C}^{\prime}$ be a regular Cauchy structure on $X$ such that $\mathscr{C}^{\prime} \leqq \mathscr{C}$. Then $q_{\mathscr{C}} \leqq q$ implies that $q_{\mathscr{C}^{\prime}}=q$. Since $\mathscr{C}^{\prime}$ is regular, $\mathscr{D}_{q} \leqq \mathscr{C}^{\prime} \leqq \mathscr{C}^{q}$. But a minimal regular convergence space is shown in [1] to be regular-closed, and it follows from Theorem 2.2 that $\mathscr{D}_{q}=\mathscr{C}^{\prime}=$ $\mathscr{C}^{q}=\mathscr{C}$. Thus $(X, \mathscr{C})$ is a minimal regular Cauchy space.

To simplify the formulation of the next theorem, we introduce the following two condition on a Cauchy space $(X, \mathscr{C})$.

Condition A. Let $\mathscr{H}$ be an u.f. on $X$ and $a \in X$. If, for all $n$ in $N, \mathscr{F} \in \Delta_{q}$, and $y \neq a$, it is true that $\left(\Gamma_{q}^{n} \mathscr{H}\right) \vee \mathscr{F}=0$ and $\left(\Gamma_{q}^{n} \mathscr{H}\right) \vee \mathscr{\mathscr { V }}_{q}(y)=0$, and it is also true that $\mathscr{G} \vee\left(\Gamma_{q}^{m} \mathscr{H}\right) \neq 0$ for some $\mathscr{G} q$-converging to $a$ and $m \in N$, then $\mathscr{H} q$-converges to $a$.

Condition B. There are at least two filters $\mathscr{F}$ and $\mathscr{G}$ on $X$ which $q$-converge to distinct points such that $\mathscr{F} \vee \mathscr{K}_{q} \neq 0$ and $\mathscr{G} \vee \mathscr{M}_{q} \neq 0$.

THEOREM 3.2. Let $(X, \mathscr{C})$ be a noncomplete regular Cauchy space, and let $q=q_{\mathscr{B}}$. Then $(X, \mathscr{C})$ is minimal regular iff $\mathscr{C}=\mathscr{D}_{q}$ and $(X, \mathscr{C})$ satisfies Conditions $\mathrm{A}$ and $\mathrm{B}$.

Proof. Assume that $\mathscr{C}=\mathscr{D}_{q}$ and that $(X, \mathscr{C})$ satisfies the two conditions. Suppose that there is a regular Cauchy structure $\mathscr{C}^{\prime}$ on $X$ such that $\mathscr{C}^{\prime}<\mathscr{C}$. Let $p=q_{\mathscr{Q}}$. Since $\mathscr{C}=\mathscr{D}_{q}, p<q$, and so there is an u.f. $\mathscr{H}$ and a point $z$ in $X$ such that $\mathscr{H} p$-converges to $z$, but $\mathscr{H}$ fails to $q$-converge to $z$.

Case 1. $\mathscr{H} \in \Delta_{q}$. If $\mathscr{K} \in \Delta_{q}$, then $\mathscr{K} \cap \mathscr{H} \in \mathscr{D}_{p} \subset \mathscr{C}$, and Condition $\mathrm{B}$ asserts that there are filters $\mathscr{F}$ and $\mathscr{G}$ which interest $\mathscr{M}_{q}$ and converge to distinct points $a$ and $b$, respectively; it follows that $\dot{z} \geqq \Gamma_{q} \mathscr{F}$ and $\dot{z} \geqq \Gamma_{q} \mathscr{G}$. Since $z$ cannot equal both $a$ and $b$, a contradiction is obtained, and it follows that $\mathscr{H} \in \Delta_{q}$. Thus each member of $\Delta_{q}$ is a nonconvergent member of $\mathscr{C}^{\prime}$; from this it follows that $\Delta_{q} \subset \Delta_{p}$.

Case 2. $\mathscr{C} \notin \Delta_{q}$. In this case there is $n \in N, a \in X$, and $\mathscr{K} q$ converging to a such that $\mathscr{K} \vee\left(\Gamma_{q}^{n} \mathscr{H}\right) \neq 0$. It must be the case that $a=z$, otherwise $\left(X, \mathscr{C}^{\prime}\right)$ would not be regular and Hausdorff. 
Since $\left(\Gamma_{p}^{n} \mathscr{\mathscr { C }}\right) \vee \mathscr{V}_{p}(b)=0$ for all $n$ in $N$ and $b \neq z$, it follows that $\left(\Gamma_{q}^{n} \mathscr{H}\right) \vee \mathscr{V}_{q}(b)=0$ for all $n$ in $N$ and $b \neq z$. Furthermore, $\left(\Gamma_{p}^{n} \mathscr{H}\right) \vee$ $\left(\Gamma_{p}^{n} \mathscr{F}\right)=0$ for all $n \in N$ and $\mathscr{F} \in \Delta_{p}$; this, along with the previously established result that $\Delta_{q} \subset \Delta_{p}$, implies that $\left(\Gamma_{q}^{n} \mathscr{H}\right) \vee\left(\Gamma_{q}^{n} \mathscr{F}\right)=0$ for all $\mathscr{F} \in \Delta_{q}$. But then, by Condition A, $\mathscr{K} q$-converges to $z$. This contradiction establishes that $(X, \mathscr{C})$ is minimal regular.

Conversely, assume that $(X, \mathscr{C})$ is minimal regular. Obviously, $\mathscr{C}=\mathscr{D}_{q}$. Suppose that under the stipulations of Condition A, $\mathscr{H}$ fails to $q$-converge to $a$. Then the Cauchy space $\mathscr{C}^{\prime}=\mathscr{C} \cup\{\mathscr{K} \in$ $F(X): \mathscr{K} \geqq \mathscr{L} \cap\left(\Gamma_{q}^{n} \mathscr{\mathscr { C }}\right)$ for some $\mathscr{L} q$-converging to $a$ and $n$ in $\left.N\right\}$ is a regular Cauchy structure on $X$, and $\mathscr{C}^{\prime}<\mathscr{C}$. This contradiction shows that Condition A must be satisfied.

Next, we show that Condition B is satisfied. Suppose, on the contrary, that there is only one point $x$ with the property that there is a filter $\mathscr{F}$ which $q$-converges to $x$ such that $\mathscr{F} \vee \mathscr{M}_{q} \neq 0$. Define $\mathscr{C}^{\prime}=\mathscr{C} \cup\{\mathscr{H} \in F(X): \mathscr{H} \geqq \mathscr{K} \cap \mathscr{L}$, for some $\mathscr{K} q$-converging to $x$ and $\left.\mathscr{L} \in \Delta_{q}\right\}$. It is easy to verify that $\mathscr{C}^{\prime}$ is regular and $\mathscr{C}^{\prime}<\mathscr{C}$. A similar construction is possible if there is no filter $\mathscr{F} \in \mathscr{C}^{q}$ such that $\mathscr{F} \vee \mathscr{l}_{q} \neq 0$; in this case the point $x$ can be chosen arbitrarily, and $\mathscr{C}^{\prime}$ constructed as before. Thus a minimal regular Cauchy space also satisfies Condition $\mathrm{B}$, and the proof is complete.

Note that $(X, \mathscr{C})$ satisfies Condition A whenever $q_{\mathscr{C}}$ is a topology.

Corollary 3.3. Let $(X, \mathscr{C})$ be a noncomplete, regular Cauchy space such that $q=q_{\mathscr{E}}$ is a topology. Then $(X, \mathscr{C})$ is minimal regular iff $\mathscr{C}=\mathscr{D}_{q}$, and $(X, \mathscr{C})$ satisfies Condition B.

The next corollary, which follows immediately from Theorems 3.1 and 3.2 and Corollary 2.4, is a complement to the latter result.

CoRollary 3.4. Let $(X, \mathscr{C})$ be a minimal ragular Cauchy space.

(a) $(X, \mathscr{C})$ is complete iff $\left(X, q_{\mathscr{E}}\right)$ is a minimal regular convergence space.

(b) $(X, \mathscr{C})$ is totally bounded iff $\left(X, q_{\mathscr{E}}\right)$ is almost topological.

There follows an example of a maximal minimal regular Cauchy space, this is to say, a minimal regular Cauchy space whose underlying convergence structure is as fine as possible (in view of Condition B).

EXAMPLE 3.5. Let $X$ be an infinite set, $a$ and $b$ two points in $X$, and $\mathscr{F}, \mathscr{G}$ two free u.f.'s on $X$. Let $q$ be the regular topology on $X$ with neighborhood filters given by: $\mathscr{V}_{q}(a)=\mathscr{F} \cap \dot{a} ; \mathscr{V}_{q}(b)=$ $\mathscr{G} \cap \dot{b} ; \mathscr{V}_{q}(x)=\dot{x}$, for $x \neq a$ and $x \neq b . \mathscr{C}=\mathscr{C}_{q}$. It is easy to see that $(X, \mathscr{C})$ satisfies Condition B. Thus, by Theorem 1.3 and Corol- 
lary 3.3, $(X, \mathscr{C})$ is minimal regular. Note that there is no finer convergence structure on $X$ for which a compatible Cauchy structure Condition B.

The final result is an immediate consequence of Theorems 2.5 and 3.2.

CoRollary 3.6. A noncomplete minimal regular Cauchy space has no regular completion.

\section{REFERENCES}

1. D. C. Kent and G.D. Richardson, Minimal Convergence Spaces, Trans. Amer. Math. Soc., 160 (1971), 487-499.

2. - Regular completions of Cauchy spaces, Pacific J. Math., 51 (1974), 483-490.

Received December 1, 1978.

Washington State UNIVERSity

Pullman, WA 99163 



\section{PACIFIC JOURNAL OF MATHEMATICS}

\section{EDITORS}

DONALD BABBITT (Managing Editor)

University of California

Los Angeles, CA 90024

Hugo RossI

University of Utah

Salt Lake City, UT 84112

C. C. MOORE and ANDREW OGG

University of California

Berkeley, CA 94720
J. DugundjI

Department of Mathematics

University of Southern California

Los Angeles, CA 90007

R. FINN and J. MILGRAM

Stanford University

Stanford, CA 94305

\section{ASSOCIATE EDITORS}
R. ARENS
E. F. BECKENBACH
B. H. NeUmanN
F. WOLF
K. YosHIDA

\section{SUPPORTING INSTITUTIONS}

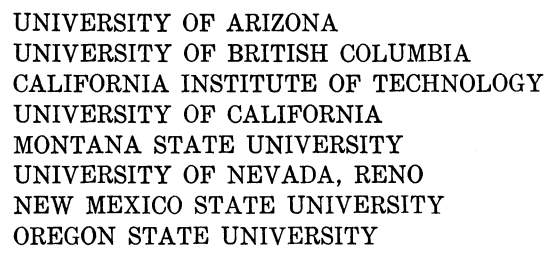

UNIVERSITY OF ARIZONA

UNIVERSITY OF BRITISH COLUMBIA CALIFORNIA INSTITUTE OF TECHNOLOGY

UNIVERSITY OF CALIFORNIA

MONTANA STATE UNIVERSITY

UNIVERSITY OF NEVADA, RENO

NEW MEXICO STATE UNIVERSITY OREGON STATE UNIVERSITY

\author{
UNIVERSITY OF OREGON \\ UNIVERSITY OF SOUTHERN CALIFORNIA \\ STANFORD UNIVERSITY \\ UNIVERSITY OF HAWAII \\ UNIVERSITY OF TOKYO \\ UNIVERSITY OF UTAH \\ WASHINGTON STATE UNIVERSITY \\ UNIVERSITY OF WASHINGTON
}

The Supporting Institutions listed above contribute to the cost of publication of this Journal, but they are not owners or publishers and have no responsibility for its content or policies.

Mathematical papers intended for publication in the Pacific Journal of Mathematics should be in typed form or offset-reproduced, (not dittoed), double spaced with large margins. Please do not use built up fractions in the text of the manuscript. However, you may use them in the displayed equations. Underline Greek letters in red, German in green, and script in blue. The first paragraph or two must be capable of being used separately as a synopsis of the entire paper. Please propose a heading for the odd numbered pages of less than 35 characters. Manuscripts, in triplicate, may be sent to any one of the editors. Please classify according to the scheme of Math. Reviews, Index to Vol. 39. Supply name and address of author to whom proofs should be sent. All other communications should be addressed to the managing editor, or Elaine Barth, University of California, Los Angeles, California, 90024.

50 reprints to each author are provided free for each article, only if page charges have been substantially paid. Additional copies may be obtained at cost in multiples of 50 .

The Pacific Journal of Mathematics is issued monthly as of January 1966. Regular subscription rate: $\$ 102.00$ a year (6 Vols., 12 issues). Special rate: $\$ 51.00$ a year to individual members of supporting institutions.

Subscriptions, orders for numbers issued in the last three calendar years, and changes of address shoud be sent to Pacific Journal of Mathematics, P.O. Box 969, Carmel Valley, CA 93924, U.S.A. Old back numbers obtainable from Kraus Per!odicals Co., Route 100, Millwood, NY 10546.

\section{PUBLISHED BY PACIFIC JOURNAL OF MATHEMATICS, A NON-PROFIT CORPORATION}

Printed at Kokusai Bunken Insatsusha (International Academic Printing Co., Ltd.). 8-8, 3-chome, Takadanobaba, Shinjuku-ku, Tokyo 160, Japan.

Copyright (C) 1981 by Pacific Jounal of Mathematics Manufactured and first issued in Japan 


\section{Pacific Journal of Mathematics}

Vol. 94, No. $2 \quad$ June, 1981

Thomas E. Armstrong and William David Sudderth, Nearly strategic

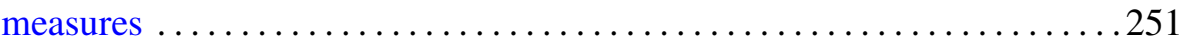

John J. Buoni, Artatrana Dash and Bhushan L. Wadhwa, Joint Browder

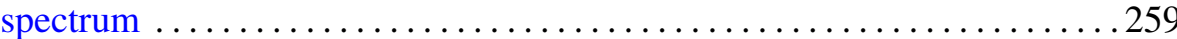

Jack Paul Diamond, Hypergeometric series with a $p$-adic variable . .......265

Raymond Frank Dickman, Jack Ray Porter and Leonard Rubin,

Completely regular absolutes and projective objects ............ 277

James Kenneth Finch, On the local spectrum and the adjoint ........... 297

Benno Fuchssteiner, An abstract disintegration theorem ............ 303

Leon Gerber, The volume cut off a simplex by a half-space $\ldots \ldots \ldots \ldots 311$

Irving Leonard Glicksberg, An application of Wermer's subharmonicity

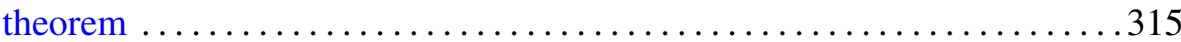

William Goldman, Two examples of affine manifolds ............... 327

Yukio Hirashita, On the Weierstrass points on open Riemann surfaces .....331

Darrell Conley Kent, A note on regular Cauchy spaces ............. 333

Abel Klein and Lawrence J. Landau, Periodic Gaussian

Osterwalder-Schrader positive processes and the two-sided Markov

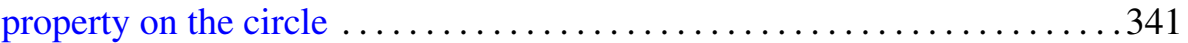

Brenda MacGibbon, $\mathscr{K}$-Borelian embeddings and images of Hausdorff

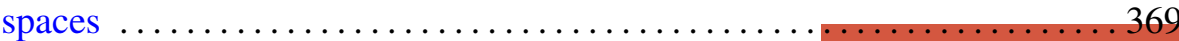

John R. Myers, Homology 3-spheres which admit no PL involutions . . . . . 379

Boon-Hua Ong, Invariant subspace lattices for a class of operators . . . . . 385

Chull Park, Representations of Gaussian processes by Wiener processes . . . 407

Lesley Millman Sibner and Robert Jules Sibner, A sub-elliptic estimate

for a class of invariantly defined elliptic systems $\ldots \ldots \ldots \ldots \ldots \ldots .417$

Justin R. Smith, Complements of codimension-two submanifolds. III.

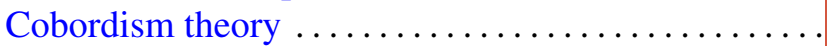

William Albert Roderick Weiss, Small Dowker spaces

David J. Winter, Cartan subalgebras of a Lie algebra and its ideals. II ... 